\title{
Atomic-scale Characterization of Restructured PtCu Nanocubes
}

\author{
Cecile S. Bonifacio ${ }^{1}$, Junjun Shan ${ }^{2}$, Franklin(Feng) Tao ${ }^{2}$, and Judith C. Yang ${ }^{1}$
}

${ }^{1 .}$ Department of Chemical and Petroleum Engineering, University of Pittsburgh, Pittsburgh, PA 15260

2. Department of Chemical \& Petroleum Engineering and Department of Chemistry, University of Kansas, Lawrence, KS 66047

Bimetallic nanoparticles are of increasing interest due to the mutual influence of different neighboring atoms that leads to catalytic behavior different than that of a monometallic cluster[1]. The catalytic properties of these materials are dependent on the catalyst atoms at the edge and/or corner[2], i.e., surface of the nanoparticle. Motivated by previous studies [3,4] resulting to surface compositional change of bimetallic catalyst through metal segregation and reconstruction; post-synthesis reaction in the gas phase with ambient pressure x-ray photoelectron spectroscopy (AP-XPS) was used to tune the composition of two types of $\mathrm{PtCu}$ nanocubes(NCs) with different surfaces. Here we have used microscopy and spectroscopy techniques to provide direct evidence of atomic-scale elemental distributions within the NCs post-synthesis gas reactions.

PtCu bimetallic NCs (8 nm in size flat shell or regular nanocubes (RNC) and $12 \mathrm{~nm}$ in size concave nanocubes (CNC)) supported in $\mathrm{Al}_{2} \mathrm{O}_{3}$ were drop-casted on holey carbon TEM gold grids. These grids were exposed to single and sequential gas reactions in $\mathrm{H}_{2}$ and $\mathrm{H}_{2}-\mathrm{CO}$ at $200{ }^{\circ} \mathrm{C}$, respectively, in situ in an AP-XPS. A FEI Titan scanning transmission electron microscope (S/TEM) with a ChemiSTEM ${ }^{\text {TM }}$ energy-dispersive $\mathrm{x}$-ray spectrometer (EDS) system operated at $200 \mathrm{KeV}$ was used to obtain the elemental distributions post-gas reactions of the NCs. The acquired EDS maps were compared with APXPS data and predicted models using periodic density functional theory (DFT) calculations of the resulting structure of the NCs before and after gas-reactions.

Figure 1 and 2 shows the EDS line scans and associated DFT models before and after the reactions for the RNCs and CNCs, respectively. After reaction with $\mathrm{H}_{2}$ at $200{ }^{\circ} \mathrm{C}$, the initial Pt skin of the RNC was retained but the structure was transformed with more $\mathrm{Pt}-\mathrm{Cu}$ alloy distributed on the surface and some $\mathrm{Cu}$ below the Pt skin. This structure is similar to the DFT results of $\mathrm{Cu}$ at the subsurface layer for the RNCs(Figure 1c). The removal of the Pt skin, change from mesa-type to spherical shape, and redistribution of the $\mathrm{Pt}$ and $\mathrm{Cu}$ into a homogeneous alloy of the RNCs occurred after exposure to $\mathrm{CO}$ (Figure 1d). On the other hand, a significant change occurred to the CNCs after the single reaction with $\mathrm{H}_{2}$ at $200{ }^{\circ} \mathrm{C}$ with the formation of a $2 \mathrm{~nm}$ Pt shell and Pt-Cu alloy core (Figure 2c). Further reaction with $\mathrm{CO}$ resulted to an increased concentration of $\mathrm{Cu}$ at the subsurface of the CNC(Figure 2d). The increase in $\mathrm{Cu}$ signal at the subsurface concurs with the DFT prediction of $\mathrm{Cu}$ clusters/patch formation in the shell of the CNC after the sequential reaction in $\mathrm{H}_{2}-\mathrm{CO}$. The atomic elemental distributions obtained through EDS showed that the resulting surface structure and composition of the NCs depend on the gas used in the reactions[5]. The surface segregation of the bimetallic NCs drove the observed surface reconstructions during post gas reactions. Further analysis of the EDS maps, AP-XPS and DFT calculations are underway to determine the process and driving forces of surface reconstruction at the atomic level for the flat and concave nanocubes [6].

\section{References:}


[1] R. Ferrando, J. Jellinek, R.L. and Johnston, Chemical Reviews 109 (2008), p. 845

[2] J.A. Rodriguez, Surface Science Reports 24 (1996), p. 225

[3] F. Tao and M. Salmeron, Science 331 (2011), p. 171

[4] D. Hansgen et al, Nature Chemistry 2 (2010), p. 484

[5] J. Shan et al, Nature Communications (2015), Manuscript in preparation

[6] CB and JY acknowledge financial support by DOE Basic Energy Sciences (DOE-BES) and Dr.

Karen Bustillo (NCEM) for technical support. The microscopy work was done in the National Center for Electron Microscopy (NCEM), Molecular Foundry at Berkeley National Lab supported by the Office of Basic Energy Sciences of the US Department of Energy Contract No. DE-AC02-05CH11231.
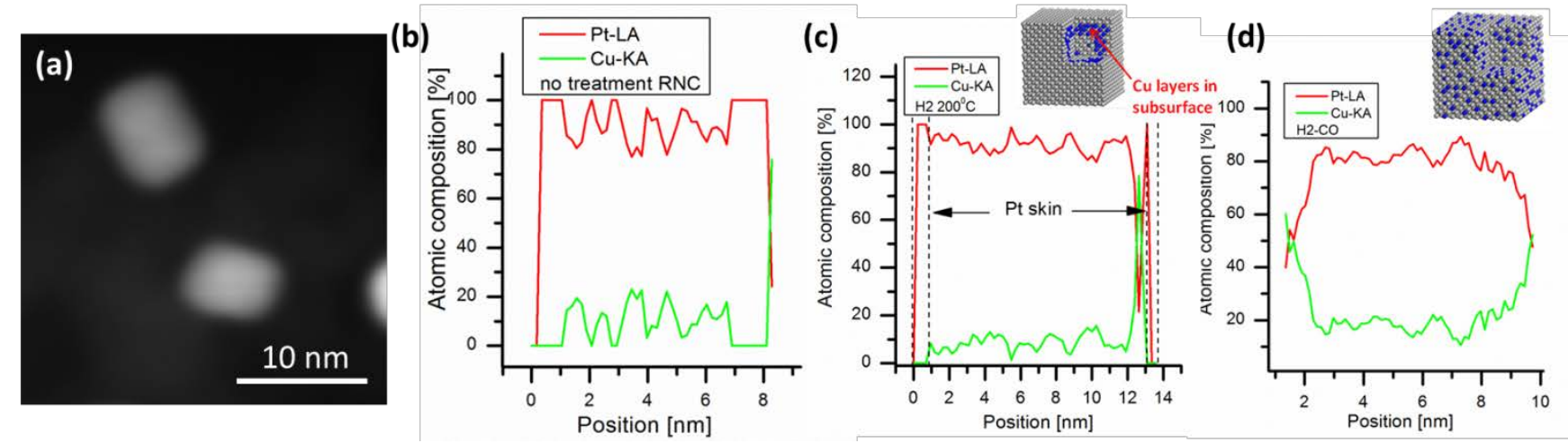

Figure 1. High angle annular darkfield (HAADF) image(a) of the RNC and EDS line scans across the $\mathrm{NC}$ before(b) and after reaction in $\mathrm{H}_{2}$ at $200{ }^{\circ} \mathrm{C}$ (c) and two sequential reactions in $\mathrm{H}_{2}-\mathrm{CO}$ (d). Gray and blue circles in the DFT models correspond to $\mathrm{Pt}$ and $\mathrm{Cu}$ atoms, respectively.
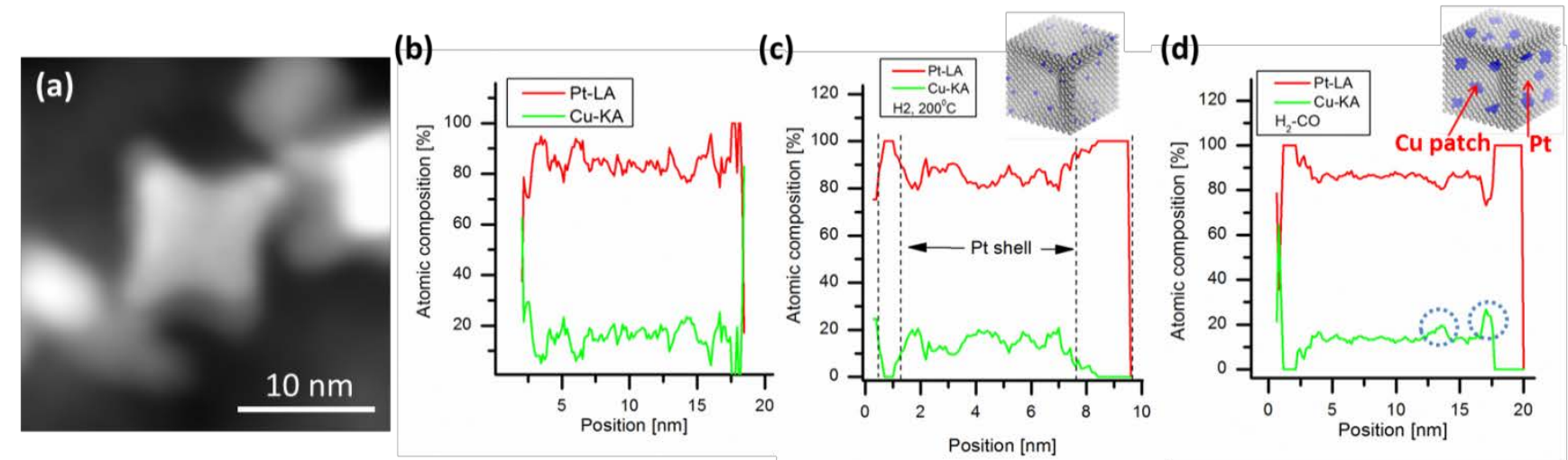

Figure 2. High angle annular darkfield (HAADF) image(a) of the CNC and EDS line scans across the $\mathrm{NC}$ before(b) and after reaction in $\mathrm{H}_{2}$ at $200{ }^{\circ} \mathrm{C}$ (c) and two sequential reactions in $\mathrm{H}_{2}-\mathrm{CO}$ (d). Gray and blue circles in the DFT models correspond to $\mathrm{Pt}$ and $\mathrm{Cu}$ atoms, respectively. 